\title{
An Algorithm of Enemy Flight Area in Fleet Air Defense
}

\author{
Long Fei ${ }^{*}$, Sun Jingdong, Tang Shaobo \\ Institute of Software and Simulation, Dalian Academy, 116018 Dalian Liaoning, China
}

\begin{abstract}
Aiming at the algorithm of the enemy flight area when the enemy launches air-to-ship missiles in fleet air defense, this paper analyzes the position situation of both parties. On the basis of the analysis, it describes the shape of the enemy flight area, and establishes the calculation model. Finally, a demonstration verification program is developed and the application examples in various cases are given. This algorithm can not only accurately calculate the enemy flight area, but effectively assist the commander to form scientific air defense plans.
\end{abstract}

\section{INTRODUCTION}

In fleet air defense, one of the main air threats for our fleet is the attack of air-to-ship missiles from the enemy aircrafts. In response to the threat, there are two modes of air defense. One mode is resisting the enemy aircrafts. Naval aviation and regional/close range air defense ships are used to intercept and resist the incoming enemy aircrafts. The other mode is resisting the hostile air-toship missiles. Regional/close range air defense ships are used to combat the incoming hostile air-to-ship missiles [1].

This paper focuses on determining the enemy flight area, which is a key issue for the mode of resisting the enemy aircrafts. Enemy flight area refers to the possible flight area for the enemy aircrafts when using air-to-ship missiles to carry out attacks. The enemy aircrafts may take off from threat sources such as airports or aircraft carriers. Our party must accurately grasp the flight area of the enemy aircrafts as preplanning the air defense and making emergency decisions, so as to carry out effective defense demand analysis and force assignment, and form scientific interception and resistance plans [2].

\section{POSITION SITUATION ANALYSIS OF BOTH PARTIES}

\subsection{Establishment of Plane Rectangular Coordinate System}

Grasping the enemy aircraft's projection on the sea surface is adequate to formulate air defense plan in practice, so the plane rectangular coordinate system $x O y$ is established. The initial condition is that the location of our fleet is Point $W$, and the enemy airport or aircraft carrier locates in Point $O$, with a distance of $d$ in between. The enemy aircraft's combat radius is $R$, and the maximum range of the enemy air-to-ship missile is $r$. So the enemy's attack distance is $R+r$. The coordinate system established is shown in Figure 1: Point $O$ is the original point, $O W$ is the Axis $x$ forward. Let's rotate $O W 90$ degrees counter clockwise and get Axis $y$. The Circle $\Omega_{1}$ is the enemy combat radius circle, with the center $O$, and the radius $R$; the Circle $\Omega_{2}$ is the enemy attack circle, with the center $O$, and the radius $R+r$; the Circle $\Omega_{3}$ is the enemy air-to-ship missile attack circle, with the center $W$, and the radius $r$.

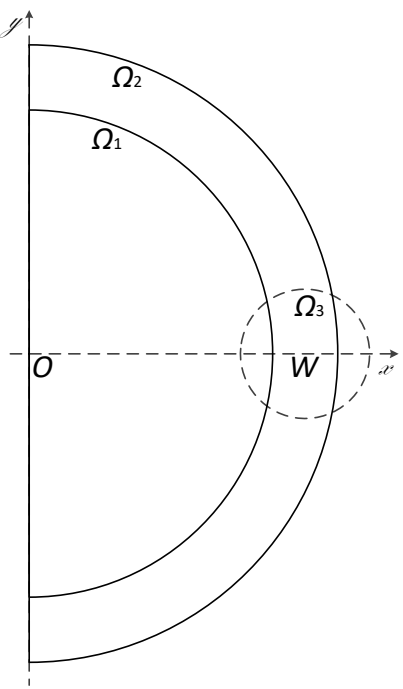

Fig. 1. Coordinate system of Position situation of both parties.

\subsection{Establishment of Plane Rectangular Coordinate System}

With the change of the distance $|O W|$ between both parties, the shapes of enemy flight area and their algorithms are divided into 3 cases. Case 1: our party locates inside the enemy attack circle, and outside the enemy combat radius circle, that is

* Corresponding author: doubao.0123@aliyun.com 
$R<|O W|<R+r$; Case 2: our party locates inside the enemy combat radius circle, and is beyond the maximum range of the enemy air-to-ship missile, that is $r \leq|O W| \leq R$; Case 3: our party locates inside the enemy combat radius circle, and is within the maximum range of the enemy air-to-ship missile, that is $|O W|<r$.

\section{ESTABLISHMENT OF CALCULATION MODEL OF ENEMY FLIGHT AREA}

\subsection{Calculation Model of Case 1}

\subsubsection{Auxiliary geometric figure}

The maximum value of the sum of the distance between the enemy aircraft's flight distance and the enemy air-toship missile is of fixed value $R+r$, so partial boundary of the enemy flight area conforms to the geometric characteristics of an ellipse ${ }^{[3-4]}$. As in Figure 2, the auxiliary Ellipse $P$ is established, the focus is $O, W$, and the length of the long axis is $R+r$. Point $A$ and Point $B$ are the intersections of Ellipse $P$ and Circle $\Omega_{1}$. Point $C$ and Point $D$ are the intersections of Ellipse $P$ and Axis $y$.

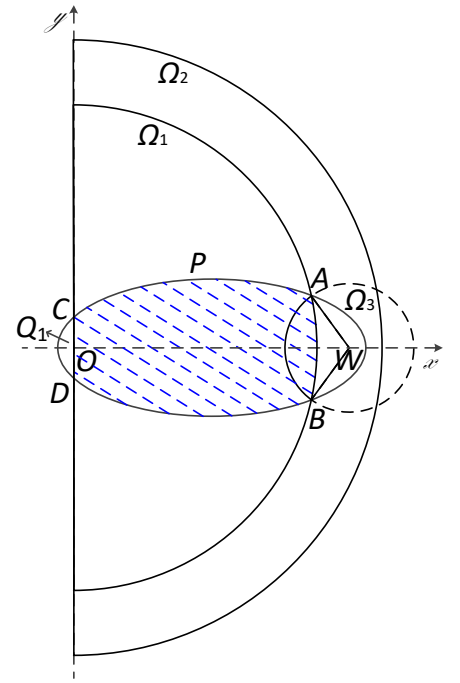

Fig. 2. Enemy flight area in Case 1.

\subsubsection{Description of the shape of the enemy flight area}

The shadow part in Figure 2 is the enemy flight area $F_{1}$, which should meet the following conditions at the same time. First, being inside or on the enemy combat radius circle $\Omega_{1}$. Second, being inside or on the Ellipse $P$. Third, the enemy aircrafts do not reverse the flight during the attack, that is, Area $Q_{1}$ is excluded. Therefore, $F_{1}$ is:

$$
F_{1}=\Omega_{1} \cap P-Q_{1}
$$

Of which $Q_{1}$ is the area formed by Segment $C D$ and $\operatorname{Arc} C D$.

Therefore, establish the equations of Circle $\Omega_{1}$ and Ellipse $P$, and calculate the coordinates of Point $A$, Point $B$, Point $C$ and Point $D$, and then, the enemy flight area $F_{1}$ can be obtained.

\subsubsection{Calculation formulas of the enemy flight area}

(1) Equation of Circle $\Omega_{1}$

$$
x^{2}+y^{2}=R^{2}
$$

(2) Equation of Ellipse $P$

$$
\frac{(x-d / 2)^{2}}{a^{2}}+\frac{y^{2}}{b^{2}}=1
$$

Of which: semi-major axis $a=(R+r) / 2$; semiminor axis $b=\sqrt{a^{2}-(d / 2)^{2}}$.

(3) Coordinates of Point $A$ and Point $B$

Point $A$ and Point $B$ are the intersection points of Circle $\Omega_{1}$, Circle $\Omega_{3}$ and Ellipse $P$, of which the equation of Circle $\Omega_{3}$ is:

$$
(x-d)^{2}+y^{2}=r^{2}
$$

The coordinates of Point $A$ and Point $B$ can be obtained from simultaneous equations of Circle $\Omega_{1}$, Circle $\Omega_{3}$ and Ellipse $P$ :

Point $A$ coordinates,

$$
\left\{\begin{array}{c}
x_{A}=\frac{d^{2}+R^{2}-r^{2}}{2 d} \\
y_{A}=\sqrt{R^{2}-\left(\frac{d^{2}+R^{2}-r^{2}}{2 d}\right)^{2}}
\end{array}\right.
$$

Point $B$ coordinates,

$$
\left\{\begin{array}{c}
x_{B}=\frac{d^{2}+R^{2}-r^{2}}{2 d} \\
y_{B}=-\sqrt{R^{2}-\left(\frac{d^{2}+R^{2}-r^{2}}{2 d}\right)^{2}}
\end{array}\right.
$$

(4) Coordinates of Point $C$ and Point $D$

Point $C$ and Point $D$ are on the Ellipse $P$. Place $x_{C}=0, x_{D}=0$ into the equation of Ellipse $P$, and the coordinates of Point $C$ and Point $D$ can be obtained:

Point $C$ coordinates,

$$
\left\{\begin{array}{c}
x_{C}=0 \\
y_{C}=b \sqrt{1-\left(\frac{d}{2 a}\right)^{2}}
\end{array}\right.
$$

Point $D$ coordinates,

$$
\left\{\begin{array}{c}
x_{D}=0 \\
y_{D}=-b \sqrt{1-\left(\frac{d}{2 a}\right)^{2}}
\end{array}\right.
$$

\subsection{Calculation Model of Case 2}

\subsubsection{Auxiliary geometric figure}


The geometric properties of Case 2 and Case 1 are basically the same, with only an area added. With the change of $|O W|$, which is the distance between the enemy and our party, the area is divided into two cases: Case 2.1: When $R-r \leq|O W| \leq R$, schematic diagram of the calculation of enemy flight area is as shown in Figure 3. Case 2.2, when $r \leq|O W|<R-r$, schematic diagram of the calculation of enemy flight area is as shown in Figure 4. An auxiliary Sector $S_{O A_{1} B_{1}}$ is established. Straight line $O A_{1}$ and $O B_{1}$ are tangent to Circle $\Omega_{3}$, intersect Circle $\Omega_{1}$ respectively at Point $A_{1}$ and Point $B_{1}$, and intersect Ellipse $P$ respectively at Point $C_{1}$ and Point $D_{1}$.

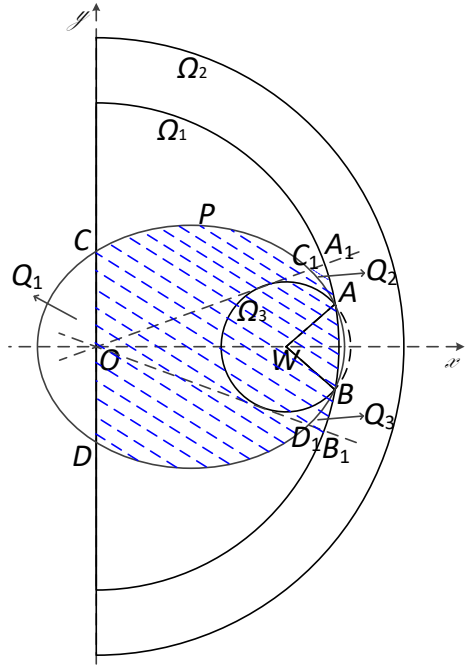

Fig. 3. Enemy flight area in Case 2.1.

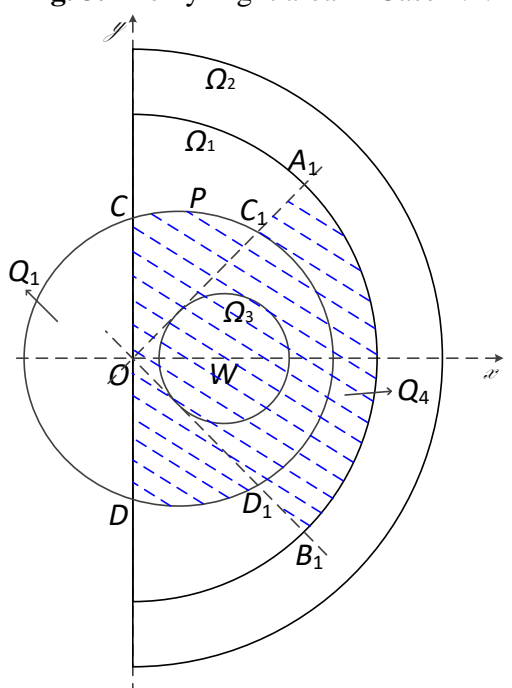

Fig. 4. Enemy flight area in Case 2.2.

\subsubsection{Description of the shape of the enemy flight area}

The shadow parts in Figure 3 and Figure 4 are the enemy flight area $F_{2}$, that is , on the basis of $F_{1}$, Sector $S_{O A_{1} B_{1}}$ is superimposed. Therefore, $F_{2}$ is :

$$
F_{2}=\left(\Omega_{1} \cap P-Q_{1}\right) \cup S_{O A_{1} B_{1}}
$$

In fact: in Case 2.1, $F_{2}$ has an additional area of $Q_{2} \cup Q_{3}$ relative to $F_{1}$, wherein $Q_{2}$ is the area enclosed by Segment $A_{1} C_{1}, \operatorname{Arc} A C_{1}$ and $\operatorname{Arc} A A_{1}$, and $Q_{3}$ is the area enclosed by Segment $B_{1} D_{1}$, Arc $B D_{1}$ and Arc $B B_{1}$. In Case 2.2, $F_{2}$ has an additional area of $Q_{4}$ relative to $F_{1}$, wherein $Q_{4}$ is the area enclosed by Segment $A_{1} C_{1}$, Segment $B_{1} D_{1}$, Arc $A_{1} B_{1}$ and Arc $C_{1} D_{1}$.

Therefore, on the basis of the algorithm of Case 1, calculate the coordinates of Point $A_{1}$, Point $B_{1}$, and then, the enemy flight area $F_{2}$ can be obtained.

\subsubsection{Calculation formulas of the enemy flight area}

Equation of Straight line $O A_{1}$ and Straight line $O B_{1}$ :

$$
\pm k x-y=0
$$

The distance between Point $F(d, 0)$ and Straight line $O A_{1}$ or Straight line $O B_{1}$ is $r$. According to this, $k=\mp \sqrt{r^{2} /\left(d^{2}-r^{2}\right)}$.

The coordinates of Point $A_{1}$ and Point $B_{1}$ can be obtained from simultaneous equations of Circle $\Omega_{1}$ :

Point $A_{1}$ coordinates,

$$
\left\{\begin{array}{c}
x_{A_{1}}=R \sqrt{1-r^{2} / d^{2}} \\
y_{A_{1}}=R r / d
\end{array}\right.
$$

Point $B_{1}$ coordinates,

$$
\left\{\begin{array}{c}
x_{B_{1}}=R \sqrt{1-r^{2} / d^{2}} \\
y_{B_{1}}=-R r / d
\end{array}\right.
$$

\subsection{Calculation Model of Case 3}

According to the rule that the enemy aircrafts do not reverse the flight during the attack, and the analysis of Case 2, in Case 3, the sector angle of Sector $S_{O A_{1} B_{1}}$ is defined as $\pi$, and $S_{O A_{1} B_{1}}$ properly include $\Omega_{1} \cap P-Q_{1}$.

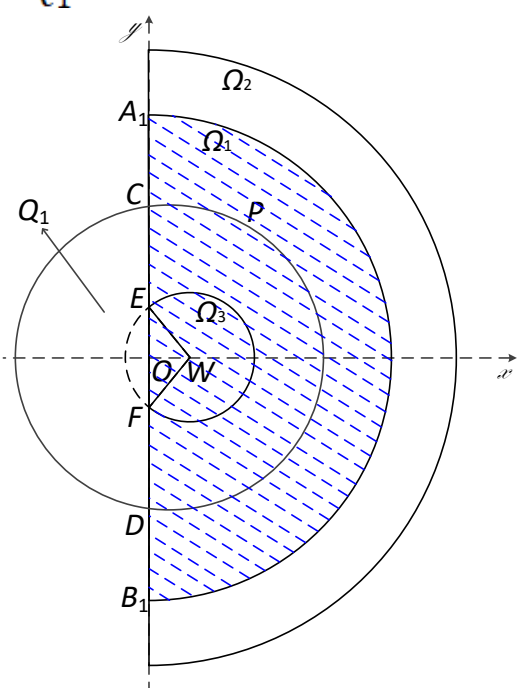

Fig. 5. Enemy flight area in Case 3. 
Therefore, $F_{3}$ is:

$$
F_{3}=S_{O A_{1} B_{1}}
$$

On the basis of the equation of Circle $\Omega_{1}$, the enemy flight area $F_{3}$ can be obtained by calculating the coordinates of Point $A_{1}$ and Point $B_{1}$ :

Point $A_{1}$ coordinates,

Point $B_{1}$ coordinates,

$$
\left\{\begin{array}{l}
x_{A_{1}}=0 \\
y_{A_{1}}=R
\end{array}\right.
$$

$$
\left\{\begin{array}{c}
x_{B_{1}}=0 \\
y_{B_{1}}=-R
\end{array}\right.
$$

\section{APPLICATION EXAMPLE OF THE CALCULATION MODEL OF THE ENEMY FLIGHT AREA}

In order to test the algorithm of enemy flight area, a demonstration verification program is developed using Qt [5-6]. It can be used to calculate the enemy flight area under various combination of combat radius of the enemy aircraft, maximum range of the enemy air-to-ship missile, and distance between both parties.

Here is a set of assumed numeric values for calculating the enemy flight areas in various cases. It is known that the position of our fleet is Point $W$; the position of the enemy airport or aircraft carrier is Point $O$; the operational radius of the enemy aircraft $R$ is $1300 \mathrm{~km}$; the maximum range of the enemy air-to-ship missile $r$ is $230 \mathrm{~km}$; assume that the distance between the enemy and our party $d$ is $1500 \mathrm{~km}, 1125 \mathrm{~km}, 600 \mathrm{~km}$ and $175 \mathrm{~km}$, respectively corresponding to Case 1 , Case 2.1, Case 2.2 and Case 3, then the enemy flight area $F_{1}, F_{2.1}, F_{2.2}, F_{3}$ are calculated, and described with shadow areas shown in Figure 6-9.

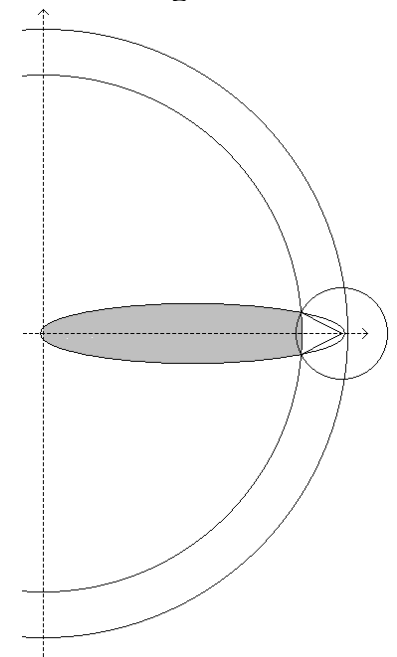

Fig. 6. Application example of enemy flight area in Case 1 $(\mathrm{d}=1500 \mathrm{~km})$.

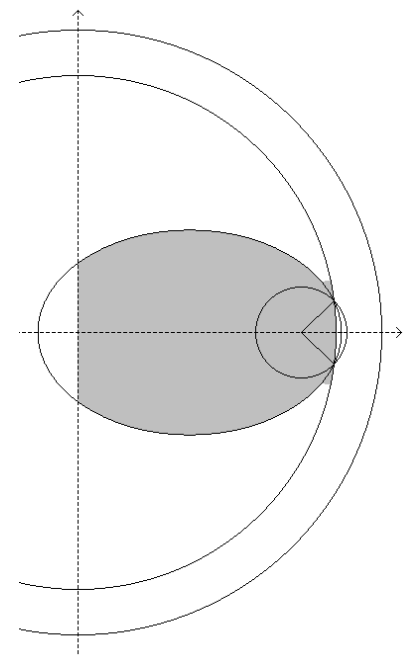

Fig. 7. Application example of enemy flight area in Case 2.1 $(\mathrm{d}=1125 \mathrm{~km})$.

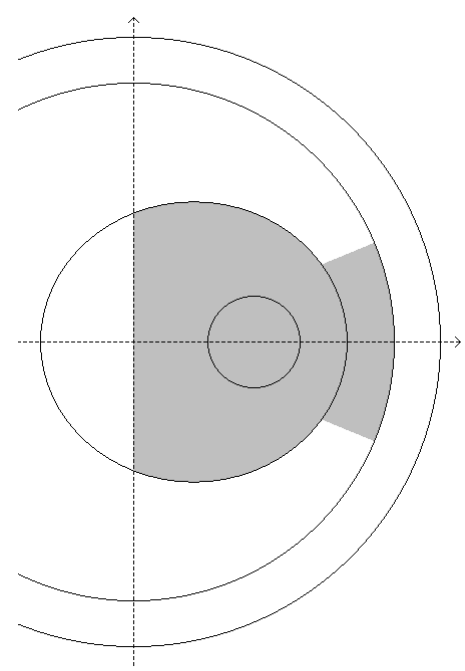

Fig. 8. Application example of enemy flight area in Case 2.2 $(\mathrm{d}=600 \mathrm{~km})$.

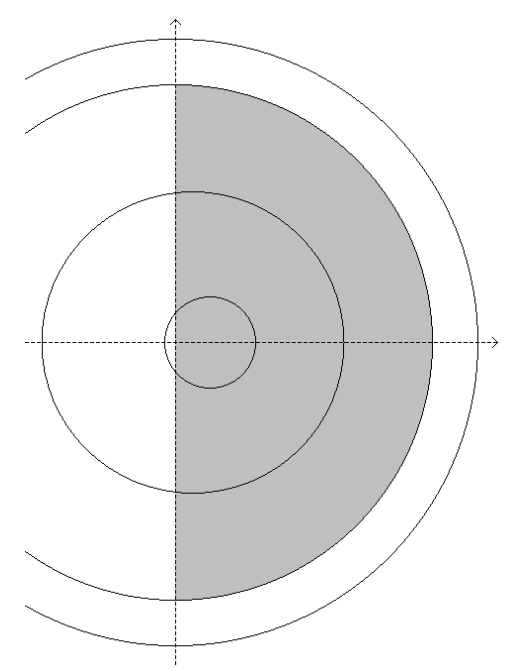

Fig. 9. Application example of enemy flight area in Case 3 $(\mathrm{d}=175 \mathrm{~km})$. 


\section{CONCLUSION}

Aiming at the algorithm of the enemy flight area when the enemy launches air-to-ship missiles with aircrafts in fleet air defense, this paper carries out military requirement analysis and classification of position situation of both parties. On this basis, it describes the shape of the enemy flight area in various cases, and establishes the calculation model. Finally, a demonstration verification program is developed and the application examples in various cases are given.

The calculation model established in this paper, which is able to accurately calculate the shape of the enemy flight area, can be directly used as the basis for the analysis of defense demand and the assignment of forces. Besides, this calculation model will become an important function point of the operational planning software to effectively assist the commander to form scientific air defense plans.

\section{References}

1. TAN Ansheng, Planning and Management Analysis for Fleet Operations of Surface Vessels (National Defense Industry Press, Beijing, 2009)

2. YANG Lujing, CHEN Zhigang, LI Yu, The Theory and Application of Combat Decision-aid (National Defense Industry Press, Beijing, 2016)

3. TAN Ansheng, Planning and Management Analysis for Shipboard Early-Warning Helicopter Operations (National Defense Industry Press, Beijing, 2017)

4. YIN Chengyi, TAN Ansheng, GUO Jianglong, Command Control and Simulation, 35, 67(2013)

5. LU Wenzhou, Qt5 Development and Application Example (Third Edition) (Electronic Industry Press, Beijing, 2017)

6. HUO Yafei, CHENG Liang, Introduction to Qt5 Programming (Beijing University of Aeronautics and Astronautics Press, Beijing, 2015) 